\title{
In Vivo Assessment of Metal Organic Framework (MOFs) for the Future use as Delivery Agents for Drugs to Treat PAH
}

\author{
Nura A Mohamed ${ }^{1}$, Rob Davies ${ }^{2}$, Paul D. Lickiss ${ }^{2}$, Gemma Freeman ${ }^{2}$, Daniel Morales-Cano ${ }^{3}$, Bianca \\ Barreira $^{3}$, Nicholas S. Kirkby ${ }^{1}$, Laura Moreno ${ }^{3}$ and Jane A Mitchell ${ }^{1}$ \\ ${ }^{1}$ National Heart and Lung Institute. \\ Imperial College of London, SW3 6LY, London, United Kingdom. \\ nura.abdi11@imperial.ac.uk; n.kirkby@imperial.ac.uk; j.a.mitchell@imperial.ac.uk \\ ${ }^{1}$ Qatar Foundation Research and Development Division. \\ Qatar Foundation, 5825, Doha, Qatar. \\ nura.abdi11@imperial.ac.uk \\ ${ }^{2}$ Department of the Chemistry. \\ Imperial College of London, SW7 2AZ, London, United Kingdom. \\ r.davies@imperial.ac.uk; p.lickiss@imperial.ac.uk; gemma.freeman@imperial.ac.uk \\ ${ }^{3}$ Department of Pharmacology, School of Medicine. \\ University Complutense of Madrid, Instituto de Investigación Sanitaria Gregorio Marañón (IiSGM), Ciber de \\ Enfermedades Respiratorias (CIBERES), Madrid, Spain. \\ danmorca@gmail.com; biancabarreira@med.ucm.es; lmorenog@med.ucm.es
}

\section{Extended Abstract}

Pulmonary arterial hypertension (PAH) is a progressive, debilitating and fatal condition with no cure. However small molecule drugs, including sildenafil, selexipag and bosentan, have been developed and are used to treat PAH but do not offer a cure and life expectancy, even on medication, is only between 2-5 years after diagnosis. All PAH drugs are ubiquitous dilators and their therapeutic dose is severely limited by their systemic side-effects [1]. We are working on the hypothesis that current PAH-drugs could be used more effectively at higher local (to the lung) concentrations if delivered selectively using a nanomedicine approach. We suggest that if toxicological limitations of nanomedicines can be overcome their use in PAH to delivery current drugs to the lung could turn this fatal disease to a chronic drug-managedcondition. One nanoparticle that we are interested in is from the Materials of Institute Lavoisier (MIL), the so called MIL-89. MIL-89 is an iron based metal organic framework (MOF). MIL-89 is a good candidate for delivery of drugs because (i) it can be tailored to accommodate different drugs including those with the molecular weights of current PAH-medications (MW; 300-500) [2], (ii) it is biocompatible and biodegradable [3]; (iii) it has a large internal surface area and high drug loading capacity; (iv) it is thermally and mechanically stable; and (v) it promises a long drug release-period with the ability to incorporate different functional groups [2,4-5]. We have previously shown that MIL-89 at concentrations up to $10 \mu \mathrm{g} / \mathrm{ml}$ is non-toxic to human lung cells including those from patients with PAH. In the current study we have investigated the effects of MIL-89 in rats in vivo for markers of toxicity. MIL-89 at $50 \mathrm{mg} / \mathrm{kg}$ was administered (i.p.) for two weeks at days $0,1,3,7,10$ and 14. At each time point rats were weighed, killed, plasma and tissues collected. MIL-89 had no effect on body weight, lung oedema or on plasma markers of organ failure. Importantly, histological analysis showed that MIL-89 reached our target organ of interest, the lung. These observations are consistent with our previous in vitro studies showing that MIL-89 is non-toxic in cellular models relevant to PAH and suggest that this particular MOF is tolerated short term in vivo and accumulates in lung tissue and that this work with MOFs will offer interests to the researchers in the field of drugdelivery development using nanotechnology.

\section{References:}

[1] S. L. Archer, et al., "Basic science of pulmonary arterial hypertension for clinicians: new concepts and experimental therapies," Circulation, vol. 121, no. 18, pp. 2045-66, 2010. 
[2] P. Horcajada, et al., "Porous metal-organic-framework nanoscale carriers as a potential platform for drug delivery and imaging," Nat. Mater., vol. 9, no. 2, pp. 172-8, 2010.

[3] R. C. Huxford, et al., "Metal-organic frameworks as potential drug carriers," Curr. Opin. Chem. Biol., vol. 14, no. 2, pp. 262-8, 2010.

[4] G. Ferey, et al., "A chromium terephthalate-based solid with unusually large pore volumes and surface area," Science, vol. 309, no. 5743, pp. 2040-2, 2005.

[5] P. Horcajada, et al., "Metal-organic frameworks as efficient materials for drug delivery," Angew. Chem. Int. Ed. Engl., vol. 45, no. 36, pp. 5974-8, 2006. 